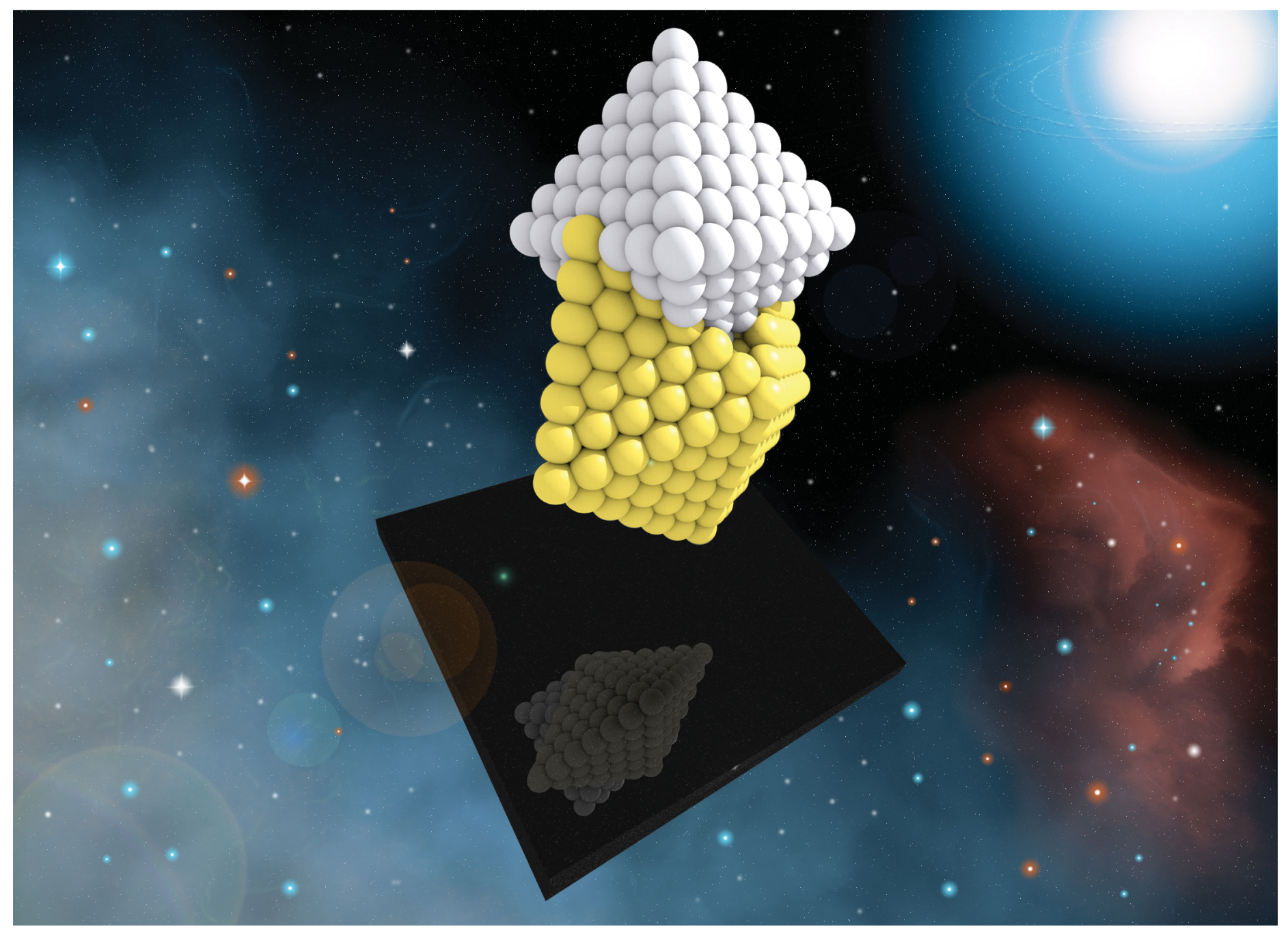

Showcasing research from the Instituto de Ciencia de Materiales de Sevilla (Spain) and the University of Cambridge (UK), funded within the framework of the Al-Nanofunc European project: "Advanced Laboratory for the Nano-analysis of Novel Functional Materials"

Title: Shape-defined nanodimers by tailored heterometallic epitaxy

Structuring nanodimer configuration is an area of great interest in the design of materials with multifunctional properties. This study shows how to use polyhedral Pt nanocrystals (cubeoctahedra, octahedra and octapods) as building blocks for the rational construction of $\mathrm{Au}-\mathrm{Pt}$ nanodimers that demonstrate epitaxial relationships such as $\{111\}+\{111\},\{200\}+\{200\}$ and $\{220\}+\{220\}$. This study enhances our understanding of heterometallic dimer formation and the well-defined nanostructures obtained promise to be of potential interest in catalysis and plasmonics.

\section{As featured in:}

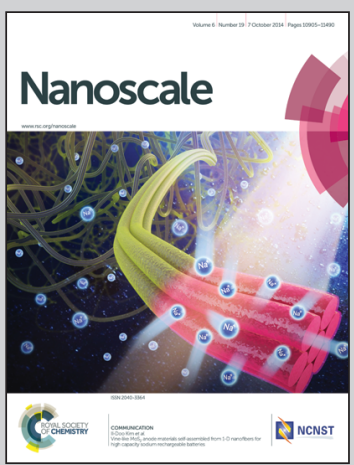

See Carlos A. García-Negrete et al., Nanoscale, 2014, 6, 11090. 


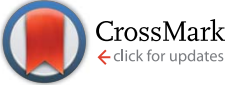

Cite this: Nanoscale, 2014, 6, 11090

\title{
Shape-defined nanodimers by tailored heterometallic epitaxy $\dagger$
}

\author{
Carlos A. García-Negrete, ${ }^{\star a}$ Teresa C. Rojas, ${ }^{a}$ Benjamin R. Knappett, ${ }^{\text {b }}$ \\ David A. Jefferson, ${ }^{b}$ Andrew E. H. Wheatley ${ }^{b}$ and Asunción Fernández ${ }^{\mathrm{a}}$
}

\begin{abstract}
The systematic construction of heterogeneous nanoparticles composed of two distinct metal domains (Au and $\mathrm{Pt}$ ) and exhibiting a broad range of morphologically defined shapes is reported. It is demonstrated that careful Au overgrowth on Pt nanocrystal seeds with shapes mainly corresponding to cubeoctahedra, octahedra and octapods can lead to heterometallic systems whose intrinsic structures result from specific epitaxial relationships such as $\{111\}+\{111\},\{200\}+\{200\}$ and $\{220\}+\{220\}$. Comprehensive analysis shows also that nanoparticles grown from octahedral seeds can be seen as comprising of four $\mathrm{Au}$ tetrahedral subunits and one Pt octahedral unit in a cyclic arrangement that is similar to the corresponding one in decahedral gold nanoparticles. However, in the present case, the multicomponent system is characterized by a broken five-fold rotational symmetry about the [011] axis. This set of bimetallic dimers could provide new platforms for fuel cell catalysts and plasmonic devices.
\end{abstract}

Received 3rd April 2014

Accepted 11th June 2014

DOI: $10.1039 / c 4 n r 01815 j$

www.rsc.org/nanoscale electrocatalytic bifunctionality, ${ }^{8}$ but also for their ability to be potentially used as enhanced nanocatalysts towards preferential carbon monoxide oxidation in the presence of hydrogen ${ }^{9}$ as well as in formic acid oxidation. ${ }^{\mathbf{1 0}}$ These applications are highly relevant in fuel cell technology. Considering the above, Au-Pt HDs with nanosized dimensions represent model systems where the goal of structurally rationalizing them is needed in order to pave the way for further application development. Constructing such hybrid structures requires pre-made particles to fuse together or, most frequently, one particle to nucleate directly onto the surface of another. ${ }^{11,12}$ After the pioneering study leading to the first generation of $\mathrm{Au}-\mathrm{Pt} \mathrm{HDs},{ }^{13}$ other studies have reported similar dimerisation processes based on direct nucleation and growth of the Au domain on Pt seeds. ${ }^{\mathbf{1 4 - 1 6}}$ However, achieving control over the spatial distribution of the two metal components into dimensionally and morphologically defined shapes remains a significant challenge. Moreover, a complete understanding of the process of heterometallic epitaxy is yet to be achieved. To make this possible, a strategy is needed for systematically dimerising $\mathrm{Au}$ and $\mathrm{Pt}$ that enables both crystallographic design and a comprehensive structural characterisation. Herein, starting from three different types of Pt seed with shapes corresponding to cubeoctahedra, octahedra and octapods, we realize the systematic construction of high yield $\mathrm{Au}-\mathrm{Pt}$ dimers that share a great variety of plane epitaxy. Based on a careful structural analysis, we identify the spatial distribution of either element in the resultant heterostructures, confirming the anisotropic metal distribution in all cases. Furthermore, we investigate the growth mode of Au on Pt seeds. Details are revealed of fringes and plane epitaxy in HDs formed when cubeoctahedral seeds, systems characterized by $\{111\}$ and 
$\{100\}$ facets, are used. New structural and synthetic paradigms are proposed for the use of octahedral seeds that are characterized by the expression of eight $\{111\}$ preferentially exposed facets and which lead to intergrown HD systems with cyclic arrangements of building blocks. The growth of HDs sharing (220) facets of $\mathrm{Au}$ and Pt, respectively, is also demonstrated through the use of Pt octapod seeds. Structural models of the different types of HDs prepared are also presented. These results confirm that the delicate structures of metallic HDs can be conveniently modified by controlling the reaction conditions in order to exploit the morphological and compositional possibilities offered by facet-selective reactivity. This study suggests new avenues for the construction of novel shapedefined HDs for applications as catalysts as well as plasmonic devices.

\section{Experimental section}

\section{Chemicals}

Platinum(II) acetylacetonate $\left(\mathrm{Pt}(\mathrm{acac})_{2}, 98 \%\right)$ was purchased from Acros Organics and used as received. Gold(III) chloride trihydrate $\left(\mathrm{HAuCl}_{4} \cdot 3 \mathrm{H}_{2} \mathrm{O}, 99 \%\right)$, cobalt carbonyl moistened with 1-10\% hexane $\left(\mathrm{Co}_{2}(\mathrm{CO})_{8}, 90 \%\right)$, manganese carbonyl $\left(\mathrm{Mn}_{2}(\mathrm{CO})_{10}, 98 \%\right)$, oleylamine (OAm, $\left.70 \%\right)$, oleic acid (OA, 99\%), 1,2-tetradecanediol (TDD, 90\%), 1,2-dichlorobenzene (anhydrous, DCB, 99\%), chloroform (anhydrous, $\geq 99 \%$ ), hexane (anhydrous, 95\%) and 2-propanol (anhydrous, 99.5\%) were purchased from Sigma-Aldrich and used as received.

\section{Synthesis of Pt seeds and Au-Pt heterodimers}

A standard 3-neck round bottom flask equipped with a condenser and a controlled temperature oil bath was used. Prior to synthesis, the flasks were flushed and all solutions were degassed with nitrogen.

\section{Preparation of cubeoctahedral Pt seeds}

The preparation of cubeoctahedral Pt seeds was based on a previous report ${ }^{17}$ with some modifications. Briefly, a solution containing $450 \mu \mathrm{L}$ of OAm, $83 \mathrm{mg}$ of TDD and $10 \mathrm{~mL}$ of DCB was added to the flask at room temperature under nitrogen and with continuous stirring. $3 \mathrm{~mL}$ of a solution of $40 \mathrm{mg}$ of $\mathrm{Pt}(\mathrm{acac})_{2}$ $(0.10 \mathrm{mmol})$ in DCB was then added immediately and the temperature was slowly increased over $\sim 35 \mathrm{~min}$ to $180{ }^{\circ} \mathrm{C}$. At this temperature, $100 \mu \mathrm{L}$ of DCB containing a trace amount of $\mathrm{Co}_{2}(\mathrm{CO})_{8}(2 \mu \mathrm{mol})$ was immediately added to the reaction medium. The resulting solution was then boiled for $30 \mathrm{~min}$ more before being gradually cooled to room temperature. After washing with $50 \mathrm{~mL}$ of $\mathrm{CHCl}_{3}$-isopropanol $1: 4$, the Pt seeds were redispersed in $10 \mathrm{~mL}$ of hexane for further studies.

\section{Preparation of octahedral and octapodal Pt seeds}

The synthesis was performed according to ref. 18. Briefly, a precursor mixture was prepared by dissolving $0.08 \mathrm{~g} \mathrm{Pt}(\mathrm{acac})_{2}$ in $10 \mathrm{~mL}$ of benzyl ether, $7.36 \mathrm{~mL}$ of OAm and $1.25 \mathrm{~mL}$ of OA under $\mathrm{N}_{2}$ atmosphere. An additive solution of $8 \mathrm{mg}$ of $\mathrm{Mn}_{2}(\mathrm{CO})_{10}$ in $1 \mathrm{~mL}$ of chloroform was then injected (rapidly) into the precursor mixture at $160{ }^{\circ} \mathrm{C}$, and the resulting mixture was heated to $220^{\circ} \mathrm{C}$. After $30 \mathrm{~min}$, the solution was cooled and octahedral nanocrystals were isolated by adding ethanol or isopropanol followed by centrifugation. The obtained product was redispersed in hexane for further use. For octapod synthesis, the procedure was the same as that described above except that the additive solution was $4 \mathrm{mg}$ of $\mathrm{Mn}_{2}(\mathrm{CO})_{10}$ in $4 \mathrm{~mL}$ of benzyl ether and the injection temperature was $180^{\circ} \mathrm{C}$.

\section{Preparation of Au-Pt heterodimers}

The procedure for the growth of Au-Pt heterodimers is based on modified literature methods. ${ }^{15,16}$ First, a precursor mixture was prepared by mixing $0.1 \mathrm{~g}$ of $\mathrm{HAuCl}_{4} \cdot 3 \mathrm{H}_{2} \mathrm{O}$ and $2 \mathrm{~mL}$ of OAm with $20 \mathrm{~mL}$ of chloroform at room temperature under an $\mathrm{N}_{2}$ atmosphere. After mixing, the previously prepared Pt seed was immediately added and the resulting media were stirred for different time intervals at a defined temperature. When cubeoctahedral Pt seeds were used, the dimerisation process was followed at room temperature for 48 hours, while in the cases where octahedral and octapodal seeds were used, the reaction temperature was $60{ }^{\circ} \mathrm{C}$ and the $\mathrm{Au}$ overgrowth was allowed for 5 and 2 hours, respectively. The dimers were precipitated through the addition of three parts by volume of ethanol or isopropanol followed by centrifugation at $4000 \mathrm{rpm}$ for $30 \mathrm{~min}$. The obtained solid was then redispersed in hexane for further use.

\section{Characterization}

TEM images were obtained using either a Philips CM200 microscope operating at an accelerating voltage of $200 \mathrm{kV}$ or a JEOL JEM-3011 HRTEM operating at $300 \mathrm{kV}$. HAADF-STEM, EDX mapping, and EDX analyses were performed using an FEI Tecnai G2F30 S-Twin STEM microscope, operated at $300 \mathrm{kV}$, with $0.2 \mathrm{~nm}$ point resolution, and equipped with a HAADF Fischione detector with $0.16 \mathrm{~nm}$ point resolution, and an INCA X-Max 80 silicon drift detector (SDD) for EDX. Gatan Digital Micrograph software was used to acquire images and perform further image processing. The diffraction tool plug-in (developed in Cadiz University) for Digital Micrograph software was used to obtain the digital diffraction patterns (fast Fourier transform) and to measure the distances and angles between the planes. The programs EjeZ and Rhodius from the University of Cadiz (Perez-Omil, 1994) ${ }^{19}$ were used to simulate the digital diffraction patterns and to construct hard sphere models of the crystalline lattices, respectively. For the tomography studies, a single tilt series of HAADF-STEM images was recorded over a wide angular range (from $-70^{\circ}$ to $+70^{\circ}$ ) every $2^{\circ}$ using a Fischione Ultra-Narrow Gap tomography holder. Images were aligned sequentially using Inspect 3D. Reconstructions were performed with Inspect 3D using an iterative routine (SIRT), which constrains the reconstructed volume to best match the original images when reprojected back along the original tilt directions. Voxel projections were undertaken using Amira software. For sample preparation, 5-10 $\mu \mathrm{L}$ of dimer or seed dispersed in hexane was pipetted onto a TEM grid and left to dry in air. For the routine analyses, carbon-coated copper grids were 
used, while an ultrathin carbon/holey carbon support film on 400 mesh Cu (TedPella no. 01824) was used for the HRTEM and HAADF-STEM analyses.

\section{Results and discussion}

\section{Au-Pt HD synthesis}

A series of Au-Pt HDs was prepared using a modified seedmediated growth method employing chloroform media ${ }^{\mathbf{1 6}}$ and using oleylamine as both the reductant and capping agent. In the reaction procedure, hexane dispersions of $\mathrm{Pt}$ seeds were added to a chloroform solution of gold(III) chloride containing a fixed amount of oleylamine. Different temperatures and reaction times were used depending on the type of Pt seed (see the Experimental section). The reddish-orange dispersion adopted a near black appearance immediately after the addition of the seed to the reducing mixture. Subsequent stirring at either room temperature (for cubeoctahedral Pt seeds) or $60{ }^{\circ} \mathrm{C}$ (for octahedral and octapodal seeds) caused the reaction mixture to evolve towards a reddish colour, which is indicative of the formation of Au-Pt HDs. ${ }^{15}$ The final dispersion could be precipitated by the addition of ethanol or isopropanol followed by centrifugation. The precipitated colloids could be redispersed in non-polar solvents such as chloroform or hexane. Fig. 1 shows representative TEM images of the different Pt seeds used (cubeoctahedra, octahedra and octapod), as well as the corresponding $\mathrm{Au}$ overgrowth products. Despite the shape diversity in the Pt seeds used (Fig. 1A-C), it was clear that HDs were predominantly obtained (Fig. 1D-F). However, the preparative conditions employed for cubeoctahedra-derived HDs proved to be crucial to controlling the morphology of the resulting HDs, due to the possibility of $\mathrm{Au}$ preferentially growing on either/both of the exposed Pt $\{111\} /\{100\}$ facets. Standard preparations of cubeoctahedra-derived HDs were performed under mild temperature conditions. Specifically, Au overgrowth for 48 hours at room temperature led to binary particles (Fig. 1D), in which the two different domain sizes were measured as $4.67 \pm 0.74 \mathrm{~nm}$ and $8.39 \pm 1.4 \mathrm{~nm}$, respectively. The dimerisation yield (based on particle counting) was $\sim 85 \%$ with non-binary particles also still visible in some areas. Attempts to perform the Au overgrowth for a longer reaction time $(120 \mathrm{~h})$ did not improve the dimerisation yield (ESI, Fig. S1†). In contrast, Au overgrowth on the cubeoctahedral seeds at higher temperature $\left(60^{\circ} \mathrm{C}\right)$ resulted only in poorly
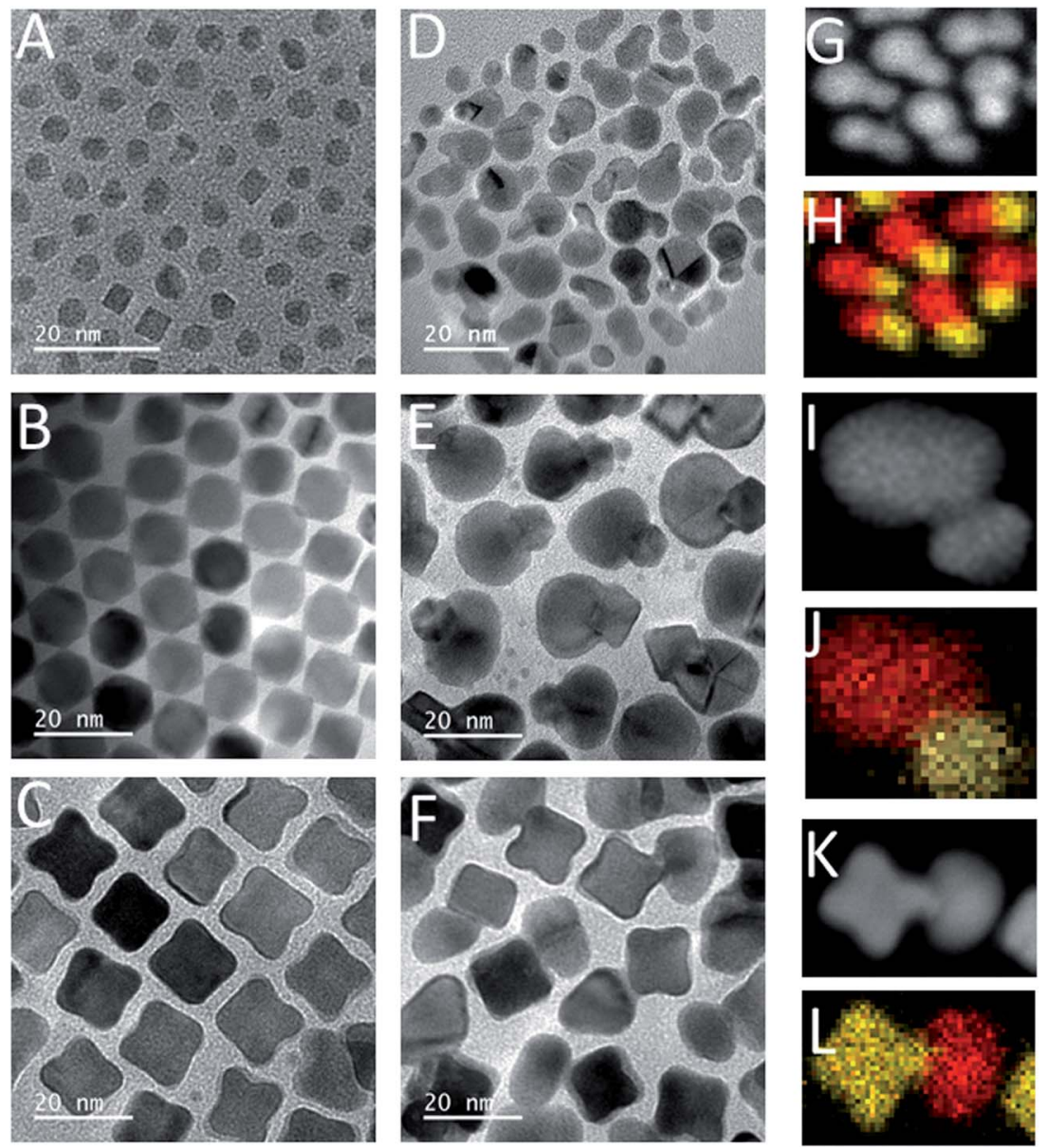

Fig. 1 Representative TEM images of (A) Pt cubeoctahedra, (B) Pt octahedra, (C) Pt octapods, and (D-F) the corresponding dimers after Au growth. (G, I and K) HAADF-STEM images of the corresponding Pt-Au dimers along with EDX chemical maps $(\mathrm{H}, \mathrm{J}$ and $\mathrm{L})$ which show the Au $(\mathrm{red})$ and Pt (yellow) distribution. 
uniform HDs, characterized by twisted shapes in some cases (ESI, Fig. S2†). Optimisation of the preparative method for cubeoctahedrally-derived HDs represents an issue to be addressed in the future as the target of the work switches to applications development. However, the present observation that some proportion $(\sim 15 \%)$ of the particles present after room temperature reduction do not demonstrate a binary structure does not detract from the main goal of the current study, that is, both to significantly extend and demonstrate the morphological versatility in the Pt-seed HD design concept and to structurally characterise and rationalise the structural diversity in the resulting library of Au-Pt HDs.

On account of phenomena such as asymmetric domain formation and variance in the inter-particle boundaries in cubeoctahedrally-seeded products, we will refer hereafter to domain average sizes instead of HD average size. In the cases of octahedral and octapodal seeding, binary particle formation was strongly effective at $60{ }^{\circ} \mathrm{C}$, leading to a yield of $\sim 100 \%$ dimer formation after 5 and 2 hours, respectively. In the corresponding octahedrally and octapodally-derived binary particles (element mapping is described below), the overgrown domains reached average sizes of $16.0 \pm 2.4 \mathrm{~nm}$ and $14.1 \pm$ $3.2 \mathrm{~nm}$, respectively, while the seed average sizes remained practically unchanged at $12.5 \pm 2.0$ and $12.3 \pm 1.6 \mathrm{~nm}$, respectively. To elucidate the compositional nature of the different domains in the binary particle types, detailed dispersive X-ray spectroscopy (EDX) analyses were carried out by means of scanning transmission electron microscopy (STEM). Fig. 1 shows the high angle annular dark field (HAADF)-STEM images (G, I and $\mathrm{K}$ ) and the relevant EDX chemical maps (H, J and $\mathrm{L}$ ) acquired by scanning an electron probe with a diameter of around $1 \mathrm{~nm}$ and registering the characteristic X-ray photons for $\mathrm{Au} L \alpha 1$ (shown in red) and Pt $\mathrm{L} \alpha 1$ (shown in yellow) from the same regions as those shown in the HAADF-STEM images. The resulting maps clearly reveal the existence of two distinct domains in each dimer, with one composed largely of gold and the other of platinum in each case. Notably, EDX analysis reveals a sharp change from Au $\mathrm{L} \alpha 1$ counts to Pt $\mathrm{L} \alpha 1$ at a distinct boundary coincident with the point of junction between the two morphological domains of each dimer. Regarding the domain shapes, based on the representative TEM images and EDX maps shown in Fig. 1, it is clear that the Pt domain is almost unchanged relative to the original seed, while that which comprises the Au differs significantly between HD types. This suggests that the Au growth mode is strongly influenced by the seed type and implies the ability to tailor the parameters of the Au domain. As a result, a comprehensive analysis of a large number of HDs was performed by means of high resolution TEM (HRTEM) in order to identify possible epitaxial relationships between the seed particle morphology, resulting HD type, and growth pathway.

\section{HRTEM studies on Au-Pt HDs}

In the face-centred cubic (fcc) phase, the lattice mismatch for the $\{111\}$ planes between $\mathrm{Pt}$ and $\mathrm{Au}$ is $3.4 \%$, while for $\{200\}$ or $\{220\}$ planes the mismatches have been reported to be 3.9 and $3.5 \%$, respectively. ${ }^{16}$ In principle, for perfect epitaxial growth, only a few monolayers of Au should be able to grow on the seed before plastic relaxation occurs. ${ }^{13}$ Different forms of plane epitaxy between $\mathrm{Au}$ and $\mathrm{Pt}$ have indeed been observed in this study. In the following sections, based on HRTEM characterization and the nature of the seed type used, the structural diversity in the resulting library of Au-Pt HDs is discussed.

Cubeoctahedra-derived HDs. HDs prepared from cubeoctahedral Pt seeds were found to adopt two main structural types. The first HD type could be properly imaged when it is oriented along the [011] zone axis. A HRTEM image of this HD type is shown in Fig. 2A. Digital diffraction patterns (DDPs) obtained from the selected areas marked in the image are shown in Fig. 2B and C. The different spots correspond to sets of atomic planes in the crystalline structure of either element, and are labelled as such. The spacing values of $2.35 \AA$ and $2.05 \AA$ measured from the DDPs can be respectively assigned to the (111) and (200) planes of fcc Au. Meanwhile, the values of $2.26 \AA$ and $1.94 \AA$ are attributable to the (111) and (200) planes of the fcc Pt. As an example, very specific (111) spacings are also indicated in the HRTEM image (Fig. 2A). In this HD type, the $\mathrm{Au}$ portion is clearly faceted and the boundaries of five subunits
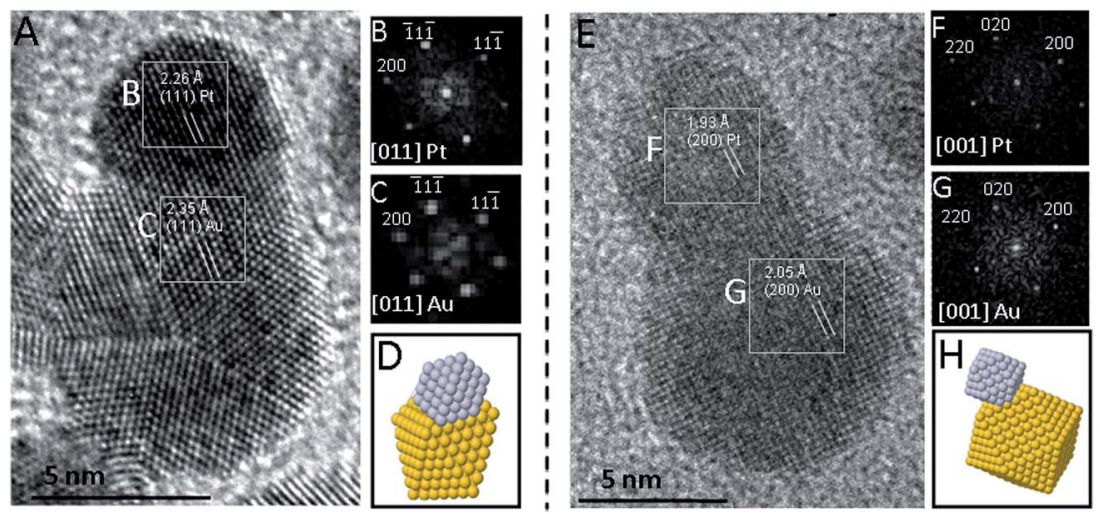

Fig. 2 Representative HRTEM images of dimer types observed from the Pt cubeoctahedrally-seeded synthesis: (A) after Au growth through (111) plane epitaxy, and (E) after Au growth through (200) plane epitaxy. (B and C, F and G) Digital diffraction patterns obtained from selected areas marked in the corresponding images. ( $\mathrm{D}$ and $\mathrm{H}$ ) Schematic hard sphere models of either dimer type. 
with fcc crystal structure and $\{111\}$ facets are observed. These subunits share a common edge coinciding with a non-crystallographic five-fold rotation axis such as is normally expected in decahedral (Dh) Au particles. ${ }^{20}$ A prominent structural feature of these is that one of the five facets of the Dh particle maintains an epitaxial relationship with the Pt seed located at the image top through (111) plane epitaxy. As shown in Fig. 2D, a hard sphere model could depict this HD type where a Dh Au particle epitaxially grows on a cubeoctahedral Pt particle. We therefore recommend attributing the name "Dh particle-containing HD" to this HD type. Because a cubeoctahedron is an Archimedean solid with eight $\{111\}$ and six $\{100\}$ preferentially exposed facets, it follows that Au overgrowth leading to a simple two domain structure can be performed on either one of the two types of seed facet. It is, therefore, to be expected that a second HD type can be also produced from cubeoctahedral Pt seeds. This is evidenced by the representative HRTEM image shown in Fig. 2E. Additionally, DDPs (Fig. 2F-G) obtained from the selected areas marked in the image show that both crystalline sites are well oriented along the [001] zone axis. The values of $2.05 \AA$ and $1.93 \AA$ are attributable to the (200) planes of fcc $\mathrm{Au}$ and fcc Pt, respectively. These planes appear to maintain an epitaxial relationship as shown in Fig. 2E. A hard sphere model of this HD type is depicted in Fig. $2 \mathrm{H}$. It has been constructed by considering an Au cubeoctahedral particle growing on a Pt seed in the $\langle 020\rangle$ direction, orthogonal to the (200) plane. We suggest naming this HD type "Orthogonally grown HD".

Octahedra-derived HDs. Octahedral nanocrystals represent interesting solids bounded by eight preferentially exposed $\{111\}$ facets. Despite the theoretical expectation of the diverse range of particle structures generated through the $\mathrm{Au}$ epitaxial overgrowth of Pt octahedra, the vast majority of the octahedral nanocrystalline Pt seeds afford a particular HD type under the synthetic conditions used. A representative HRTEM image of this HD type is shown in Fig. 3A. The structural assembly is oriented along the [011] zone axis. In spite of the complexity of the structure, the boundaries between the Pt and Au domains

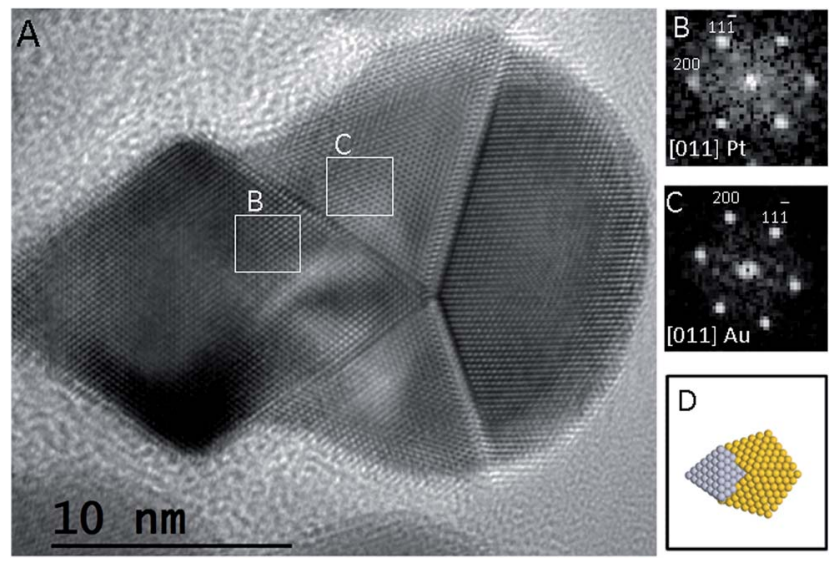

Fig. 3 (A) Representative HRTEM image of the HD type observed after Au growth on an octahedral Pt seed. (B and C) Digital diffraction patterns obtained from the selected areas marked. (D) Schematic hard sphere model of the dimer type. are distinguishable. The octahedral Pt nanocrystal has clearly retained its basic shape with two adjacent facets that share a common edge delimiting a "V"-shaped interface between the heterometallic domains. Both (111) and (200) crystallographic planes are revealed by the DDPs corresponding to boxes B and $\mathrm{C}$ (Fig. 3B and C), and these are planes which exhibit the orientations necessary to sustain epitaxial relationships between the $\mathrm{Au}$ and Pt domains. It is also noteworthy that the Au domain has a structure closely related to that of a Dh particle, as revealed by Fig. 3A. To the best of our knowledge, there exist no prior reports of $\mathrm{Au}-\mathrm{Pt} \mathrm{HDs}$ that demonstrate the conjunction of decahedral and octahedral particles. In fact, the plane epitaxy conditions that pertain between the Au and Pt domains (which demonstrate a "V"-shaped interface, see above) disturb the fivefold faceting expected for a perfectly $\mathrm{Dh} \mathrm{Au}$ domain. The result is that the whole HD in fact comprises a Au domain composed of four tetrahedral subunits fused to a single, octahedral Pt domain in an arrangement that mimics a Dh particle and which is depicted schematically in Fig. 3D. This model explains how five crystallographic building blocks can be placed in a cyclic arrangement, intersected by the juxtaposition of their exposed $\{111\}$ facets. The resulting HD type can be described as being "modified five-fold twin-defined". For the decahedral arrangement of five perfect fcc tetrahedral subunits, the angle between the adjacent $\{111\}$ facets will be $70.53^{\circ}$, which results in a $7.35^{\circ}$ solid angle deficiency. ${ }^{20}$ The result of this discrepancy is that real Dh nanoparticles must contain defects or be intrinsically strained. ${ }^{20}$ We hypothesize that the modified five-fold twin defined HDs are sufficiently stabilized through deformation. The resultant overcoming of the solid angle deficiency allows this new HD type to enrich the current debate about strain fields in decahedral particles, where new insights about internal particle structure are still emerging. ${ }^{20}$ This new HD type can be rationalised in terms of the preferential growth of $\mathrm{Au}$ nanocrystals on each of two opposite (111) facets of the Pt octahedron. A similar growth mode has been previously reported for the evolution of Ag domains on a specific number (one, two, four or five) of facets of an octahedral Pd seed. ${ }^{12}$ In this vein, HDs comprising two Au domains linked by one octahedral Pt domain were also observed in the present work (one such example is highlighted in the ESI, Fig. S3†). Icosahedrallyderived HDs were also observed in the same synthesis (one such example is also highlighted in the ESI, Fig. S4 $\dagger$ ). The presence of these HDs is not surprising as the icosahedral particles are inherent to the octahedral seed sample as shown in Fig. 1B. Both octahedral and icosahedral particles are bounded exclusively by $\{111\}$ facets, so it follows that the presence of icosahedral seeds in addition to octahedral ones will result in $\mathrm{Au}$ overgrowth on the $\{111\}$ Pt facets of both. In a model icosahedron, twenty $\{111\}$ facets are exposed in comparison with the eight $\{111\}$ facets of an octahedron. The larger number of $\{111\}$ facets per seed particle, as well as the presence of an Au domain, means that the acquisition of properly oriented HRTEM images of icosahedrally-derived HDs is not trivial. A representative HRTEM image of this HD type is included in the ESI (Fig. S4 $\dagger$ ).

Octapod-derived HDs. Pt octapods represent a nanocrystal type with a stepped surface consisting of $\{100\}$ terraces and 


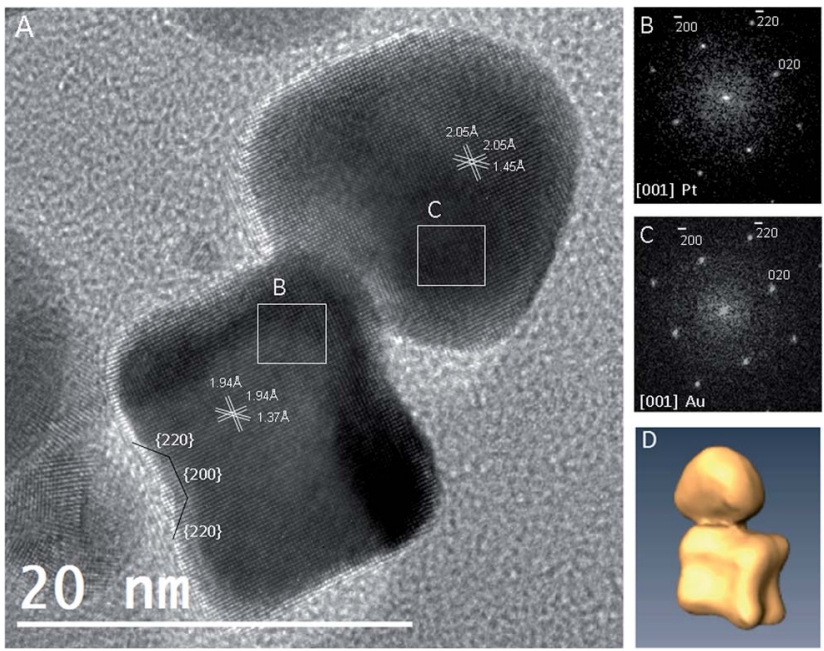

Fig. 4 (A) Representative HRTEM image of the dimer type observed after Au growth on a Pt octapod. (B and C) Digital diffraction patterns obtained from selected areas marked in the HRTEM image. (D) Volume rendering of the reconstruction obtained by means of electron tomography.

$\{110\}$ steps. ${ }^{21}$ Fig. 4A shows a representative HRTEM image of an octapod-derived HD. The image suggests that the two lattices in this HD are epitaxially oriented along the [001] zone axis, and this is corroborated by the indexed DDPs shown in Fig. 4B and C. The measured lattice fringes for the (200) and (220) planes were 2.05 and $1.45 \AA$, respectively, in the Au domain and 1.94 and $1.37 \AA$, respectively, in the Pt domain. It is interesting to note that the two domains seem to be linked through a relatively small junction. In order to gain a better understanding of the three-dimensional shape of this HD type, electron tomography was performed. In Fig. 4D (as well as in the ESI, Video S1 $\dagger$ ) the reconstructed volume and the rotation of the HD are depicted. These results show that steps located at the exposed (220) facets of the octapod seed represent the preferred sites through which to subjoin the $\mathrm{Au}$ and $\mathrm{Pt}$ domains. In other words, the $\mathrm{Au}$ domain prefers to grow from the concave edge of the seed rather than from its corner (ESI, Video $\mathrm{S} 1 \dagger$ ).

\section{Understanding the formation of Au-Pt HDs}

The characterization of the HD types reported here has confirmed that the epitaxial growth of $\mathrm{Au}$ on $\mathrm{Pt}$ can be successfully achieved in three different model systems. A schematic summarizing the results obtained in this study is shown in Fig. 5. All the dimerisation processes were performed in chloroform media by using $\mathrm{HAuCl}_{4}$ as the gold precursor and oleylamine as the capping agent. Two HD types were obtained using cubeoctahedral seeds. The first group were Dh particlecontaining and arose from preferential Au growth on one (111) facet of the nanocrystalline seed. The second type constituted orthogonally grown HDs that had their origins in preferential $\mathrm{Au}$ growth on one (200) facet of the cubeoctahedron. HD formation using cubeoctahedral seeds required the mildest conditions (room temperature and long reaction times) of all the dimerisation processes tested here. The ability to use mild

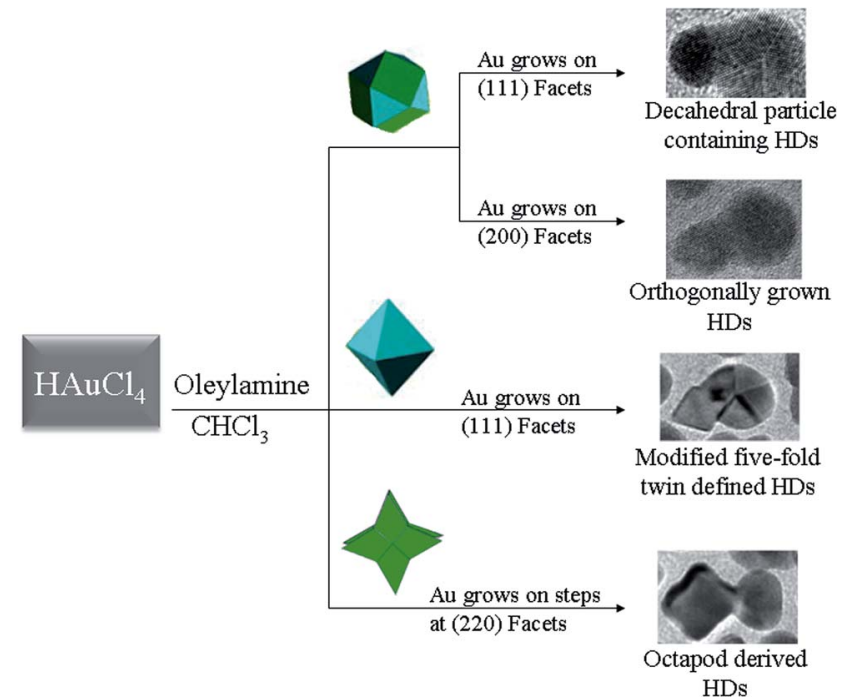

Fig. 5 Schematic summarizing the structural characterization and general synthetic steps for the formation of HDs from different Pt seeds (cubeoctahedra, octahedra and octapods).

conditions is noteworthy, since it allows the avoidance of polydispersity and twisted shapes, which would tend to form as the result of Au growing on both $\{111\}$ and $\{200\}$ Pt facets simultaneously. The growth of $\mathrm{Au}$ on two opposite (111) facets of octahedral Pt seeds was in fact observed at elevated temperatures; hence, it preferentially led to the modified five-fold twindefined HDs seen when the dimerisation process was performed at $60{ }^{\circ} \mathrm{C}$ for five hours. Octapodally-derived HDs were obtained from octapodal Pt seeds by conducting the reaction at the same temperature but for a shorter time (two hours). Overall, these observations and the experimental conditions that afforded them were consistent with the order of free energies associated with the exposed crystallographic planes: $\gamma(110)>\gamma(100)>\gamma(111){ }^{22,23}$ Due to the close proximity in free energy between the $\{200\}$ and $\{111\}$ facets, HD formation from cubeoctahedra represented the case that demanded greater levels of experimental control to avoid competing growth mechanisms. The use of room temperature, as well as a long reaction time (two days), allowed the reaction to be conducted in a selective fashion, with the Au growth being guided to either $\{200\}$ or $\{111\}$ facets of the cubeoctahedral seeds (to give either Dh particle-containing or orthogonally grown HDs, respectively) rather than occurring on both facets simultaneously. Also consistent with the order of surface free energies were the HDs formed from both octahedra and octapods. Thus, in spite of the use of similar synthetic conditions in either case, the time needed to reach a dimerisation yield of around $100 \%$ was three hours more for octahedral seeds, and this agrees well with the relative free energies associated with the $\{111\}$ and $\{220\}$ facets that are preferentially exposed in octahedra and octapods, respectively. The present study has proven a strong influence of the surface free energy associated with exposed facets with their activity in promoting the growth of Au-Pt HDs. This data also contributes to our understanding of the nucleation and growth mechanism of metallic nano-dumbbells, the most plausible 
suggestion for which was recently proposed by Shevchenko et al. ${ }^{14}$ This model suggests that: (i) surface ions $\left(e . g\right.$. $\left., \mathrm{Co}^{2+}, \mathrm{Pb}^{2+}\right)$ or $\mathrm{Co}^{2+}$ ions adsorbed at the surface of metallic seeds are required to reduce $\mathrm{Au}^{3+}$ to $\mathrm{Au}^{+}$; (ii) the nucleation event occurs as a result of the subsequent reduction of $\mathrm{Au}^{+}$by partially oxidized Pt atoms; and (iii) further growth of the gold domain then results from reduction of both $\mathrm{Au}^{3+}$ and $\mathrm{Au}^{+}$by an alkylamine at the surface of the gold nucleus. ${ }^{14}$ A further point of similarity between this thesis and the present work lies in the fact that, in both cases, growth of the Au domain is limited by species such as $\mathrm{Co}^{3+}$ and $\mathrm{Cl}^{-}$that are either present in the HD reaction solution or are formed during the synthesis of dumbbells. ${ }^{14}$ Hence, considering the similarities between the reduction approaches used by ourselves and by Shevchenko et al., ${ }^{\mathbf{1 4}}$ we take the view that Au-Pt HD formation is predicated on the continuous reduction of $\mathrm{Au}^{3+}$ throughout dimerisation. However, we suggest that while the Au nucleation step could occur randomly on the seed surface, in practice, the reduced surface energies of specific exposed facets limit the most probable sites for $\mathrm{Au}$ deposition. Further $\mathrm{Au}$ growth will then result from the reduction of both $\mathrm{Au}^{3+}$ and $\mathrm{Au}^{+}$by the alkylamine at the gold surface, as was previously proposed. ${ }^{\mathbf{1 4}}$

\section{Conclusions}

Heterometallic epitaxy has been proven herein to represent an efficient route by which to construct heterogeneous nanoparticles composed of two distinct metal domains ( $\mathrm{Au}$ and $\mathrm{Pt}$ ), affording the capability of controlling the spatial distribution of the components into dimensionally and morphologically defined shapes. The obtained colloidal hybrid nanoparticles exhibited a broad range of solid state interfaces dominated by specific epitaxial relationships such as $\{111\}+\{111\},\{200\}+$ $\{200\}$ and $\{220\}+\{220\}$. This level of sophistication, as well as the ease with which such well-defined nanostructures were produced, should be favourable to the design of noble catalysts and optical devices. An important step in the rationalization of structural diversity in the resulting library of Au-Pt HDs has also been taken. Moreover, preferentially exposed facets have been shown through this study to play a role in, and need to be considered for a better understanding of, HD formation. A more detailed study of mechanistic details is currently ongoing in our groups by means of X-ray photoelectron spectroscopy (XPS) and aberration corrected STEM analyses.

\section{Acknowledgements}

The authors gratefully acknowledge financial support from EU FP7 AL-NANOFUNC project (CT-REGPOT2011-1-285895), the Junta de Andalucía (FEDER PE2009-FQM-4554, TEP-217, PE2012-TEP862), the Spanish Ministry MINECO (CTQ201232519 ) and the CSIC (201460E018). Thanks go also to the UK Engineering and Physical Sciences Research Council for a studentship for B. R. Knappett. We acknowledge support of the publication fee by the CSIC Open Access Publication Support Initiative through its Unit of Information Resources for Research (URICI). The authors sincerely thank Dr C.
López-Cartés (Univ. Seville) for his support in the construction of hard sphere models of crystalline lattices and, Dr S. LozanoPerez (Univ. Oxford) for his advice on the STEM-EDX elemental mapping of metal nanoparticles. We also thank Mr. S. Domínguez-Meister (Instituto de Ciencia de Materiales de Sevilla) for his assistance on the back cover design.

\section{Notes and references}

1 L. Carbone and P. D. Cozzoli, Nano Today, 2010, 5, 449493.

2 C. G. Read, A. J. Biacchi and R. E. Schaak, Chem. Mater., 2013, 25, 4304-4311.

3 O. Peña Rodríguez, U. Pal, M. Campoy Quiles, L. Rodriguez Fernandez, M. Garriga and M. I. Alonso, J. Phys. Chem. C, 2011, 115, 6410-6414.

4 E. Encina and E. Coronado, J. Phys. Chem. C, 2011, 115, 15908-15914.

5 C. Liusman, H. Li, G. Lu, J. Wu, F. Boey, S. Li and H. Zhang, J. Phys. Chem. C, 2012, 116, 10390-10395.

$6 \mathrm{H}$. Yu, M. Chen, P. Rice, S. Wang, R. L. White and S. Sun, Nano Lett., 2005, 5, 379-382.

7 C. Xu, B. Wang and S. Sun, J. Am. Chem. Soc., 2009, 131, 4216-4217.

8 J. Zhu, J. Wu, F. Liu, R. Xing, C. Zhang, C. Yang, H. Yin and Y. Hou, Nanoscale, 2013, 5, 9141-9149.

9 S. Zhou, K. Mcllwrath, G. Jackson and B. Eichhorn, J. Am. Chem. Soc., 2006, 128, 1780-1781.

10 Z. Peng and H. Yang, Nano Res., 2009, 2, 406-415.

11 N. Pazos-Pérez, Y. Gao, M. Hilgendorff, S. Irsen, J. PérezJuste, M. Spasova, M. Farle, L. M. Liz-Marzán and M. Giersig, Chem. Mater., 2007, 19, 4415-4422.

12 C. Zhu, J. Zeng, J. Tao, M. C. Johnson, I. Schmidt-Krey, L. Blubaugh, Y. Zhu, Z. Gu and Y. Xia, J. Am. Chem. Soc., 2012, 134, 15822-15831.

13 T. Pellegrino, A. Fiore, E. Carlino, C. Giannini, P. D. Cozzoli, G. Ciccarella, M. Respaud, L. Palmirotta, R. Cingolani and L. Manna, J. Am. Chem. Soc., 2006, 128, 6690-6698.

14 G. Krylova, L. J. Giovanetti, F. G. Requejo, N. M. Dimitrijevic, A. Prakapenka and E. V. Shevchenko, J. Am. Chem. Soc., 2012, 134, 4384-4392.

15 S. I. Lim, M. Varon, I. Ojea-Jimenez, J. Arbiol and V. Puntes, J. Mater. Chem., 2011, 21, 11518.

16 C. Wang, W. Tian, Y. Ding, Y.-q. Ma, Z. L. Wang, N. M. Markovic, V. R. Stamenkovic, H. Daimon and S. Sun, J. Am. Chem. Soc., 2010, 132, 6524-6529.

17 S. Lim, I. Lim, M. Ojea Jiménez, E. Varon, J. Casals, J. Arbiol and V. Puntes, Nano Lett., 2010, 10, 964-973.

18 Y. Kang, J. B. Pyo, X. Ye, R. E. Diaz, T. R. Gordon, E. A. Stach and C. B. Murray, ACS Nano, 2012, 7, 645653.

19 J. A. Perez-Omil, Interpretación sistemática de imágenes de microscopía electrónica de alta resolución de materiales policristalinos. Estudiode catalizadores metálicos soportados, in Departamento de Ciencia de Materiales $e$ 
Ingenieria Metalurgica y Quimica Inorganica, University of Cadiz, Cadiz, Spain, 1994.

20 C. L. Johnson, E. Snoeck, M. Excurdia, B. RodríguezGonzález, I. Pastoriza-Santos, L. M. Liz-Marzán and M. J. Hÿtch, Nat. Mater., 2008, 7, 120-124.
21 C. Wang, C. Lin, L. Zhang, Z. Quan, K. Sun, B. Zhao, F. Wang, N. Porter, Y. Wang and J. Fang, Chem.-Eur. J., 2013, 19, 1-8. 22 Y. Sun and Y. Xia, Science, 2002, 298, 21769.

23 Z. L. Wang, J. Phys. Chem. B, 2000, 104, 1153-1175. 